\title{
A new optimization-based approach for aircraft landing in the presence of windshear
}

\author{
Paweł Drąg \\ Department of Control Systems and Mechatronics \\ Wrocław University of Science and Technology \\ Wybrzeże Wyspiańskiego 27, 50-370, Wrocław, Poland \\ Email: pawel.drag@pwr.edu.pl
}

\author{
Krystyn Styczeń \\ Department of Control Systems and Mechatronics, \\ Wrocław University of Science and Technology \\ Wybrzeże Wyspiańskiego 27, 50-370, Wrocław, Poland \\ Email: krystyn.styczen@pwr.edu.pl
}

\begin{abstract}
In the article a new approach for aircraft landing with the presence of the windshear phenomena was presented. The differential-algebraic model with variability constraints was under considerations. To transform the optimal control problem into a nonlinear optimization task, a modified direct shooting method was used. Then, to solve the obtained largescale nonlinear optimization problem, a barrier method was applied. Moreover, in the proposed optimization-based approach, the variability constraints imposed on the state trajectory were considered directly.
\end{abstract}

Index Terms-variability constraints, DAE systems, aircraft landing, barrier method.

\section{INTRODUCTION}

I $\mathrm{N}$ THE past few decades a number of significant airplane accidents have resulted from windshear, which can be observed during takeoff or landing. Especially, such meteorological and physical phenomena as convective turbulences, gust fronts and terrain-influenced can be responsible for windshear. In general, windshear cannot constitute a danger to an airplane flying, nevertheless it may have a critical impact on flight safety at low altitude. Moreover, in takeoff and landing phases, the aircraft is low above the ground and it has no much time or space to maneuver [14].

It is worth to indicate, that low-level turbulence, below 1600 $\mathrm{ft}(500 \mathrm{~m})$, cannot be directly measured but only inferred from observational data. In their work, Hon and Chan [9] have analyzed such approaches, useful to observe a terraininduced low-level turbulence intensity. There are data obtained by the scanning Doppler lidar, the terminal Doppler weather radar (TDWR), a high-resolution anemometer, as well as the operational Windshear and Turbulence Warning System (WTWS).

Recently, Chan and Hon [5] have employed a high resolution numerical aviation model (AVM) to forecast the windshear occurrence and applied it at Hong Kong International Airport to predict the terrain-induced windshear. To improve the automatic landing procedure, Zhao et al. [22] proposed a linear model of the aircraft in longitudinal motion. The applied tracking error integral equations enables us to achieve a desirable tracking performance. The presented approach has been tested on the Boeing 747 airplane and the windshear model and showed the effectiveness and robustness of the proposed approach.
The aircraft landing problem in the presence of the windshear in the context of the feedback control has been extensively investigated by Patsko et al. [16]. It was assumed, that the bounds on the wind velocity deviations from some nominal values are known, while there is no information about the windshear location and wind velocity distribution in the windshear zone. Finally, to solve the presented problem, the methods of differential game theory have been employed

- pilot (the first player) need to choose the control parameters to minimize the payoff function,

- nature (the second player) need to design an appropriate wind disturbance to obtain an opposite interest.

The mathematical formulation of an aircraft abort landing problem has been presented by Bulirsch et al. [3], [4]. The flight maneuver can be described by a minimax optimal control problem with nonlinear differential-algebraic model of the aircraft dynamics. Moreover, a first-order state constraints, as well as a control variable constraint have been imposed on the model. The obtained optimal control problem has been solved numerically using the multiple shooting approach in connection with a homotopy strategy.

One of the first flight trajectories optimization procedures in the presence of windshear have been designed by the AeroAstronautics Group of Rice University [13]. In the mentioned research three situations have been deeply investigated: takeoff, abort landing, as well as penetration landing. Then, Pytlak and Vinter [17] have presented an optimization algorithm for optimal control problems with control, state, as well as terminal constraints. The designed algorithm has been successfully applied to solve the aircraft abort landing problem in the presence of windshear.

Recently, to solve the aircraft abort landing problem in a windshear downburst, $\mathrm{Li}$ et al. [10] designed and implemented a smooth approximate function method for solving a general class of minmax optimal control problems. Finally, the necessary and sufficient optimality conditions have been derived. To solve the obtained optimal control problem the control parameterization approach, as well as a time scaling transform have been applied.

The modern numerical control procedures are designed on the basis of efficient nonlinear optimization algorithms. Especially, new variants of a barrier method are treated as 
effective procedures to solve large-scale optimization problems. Baccari et al. [1] designed a new variant of the barrier function approach for constrained Model Predictive Control (MPC) problems. Moreover, the parallelization mechanism enabled to obtain the encouraging results without increasing the computation time. A Nonlinear Model Predictive Control (NMPC) method for the trajectory tracking problem of an autonomous underwater vehicle (AUV) has been investigated by Shen et al. [18]. Then, to handle the realistic constraints on the AUV thrusters, the authors have incorporated the log barrier functions into the cost function and modified the continuation/generalized minimal residual algorithm designed by Ohtsuka [15]. Finally, the effectiveness and efficiency of the new algorithm for the AUV tracking control problem has been demonstrated. Steinboeck et al. [20] designed a new NMPC algorithm for optimization complex systems described by hyperbolic partial differential equations. Then, the discretized constrained optimal control problem has been formulated in the form of barrier functions. The model predictive control approach for linear discrete-time systems subject to state constraints has been investigated in [8]. In the on-line optimization procedure a relaxed barrier function has been implemented. Finally, both the theoretical and numerical results have been presented.

The mentioned recently designed control procedures require efficient nonlinear optimization algorithms. Therefore, according to the presented advances in Nonlinear Model Predictive Control methods, in the next sections the new optimizationbased approach for aircraft landing in the presence of the windshear is presented.

The article is constructed as follows. In Section 2 a detailed description of the problem is presented. Then, in Section 3 , the new optimization-based solving procedure with the modified direct shooting method is discussed. The results of computational simulations are presented in Section 4. Finally, the presented considerations are concluded in Section 5 .

\section{THE PROBLEM DESCRIPTION}

During the aircraft landing maneuver, the situation presented on the Fig. 1 can be observed:

1) the plane lowers the flight,

2) the speed of the aircraft is reduced,

3) the downdraft zone,

4) the strong wind from the back of the plane.

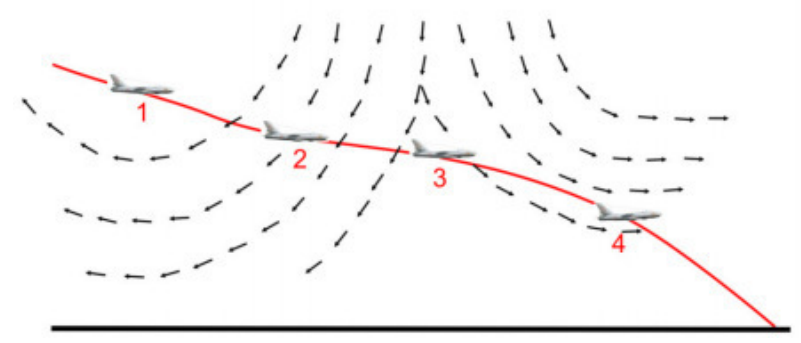

Fig. 1. The aircraft landing with the presence of the windshear [21].
The dynamical properties of the aircraft motion can be described by the following ordinary differential equations

$$
\begin{aligned}
& \dot{y}_{x}=y_{\nu} \cos y_{\gamma}+w_{x}, \\
& \dot{y}_{h}=y_{\nu} \sin y_{\gamma}+w_{h},
\end{aligned}
$$

$\dot{y}_{\nu}=\frac{1}{m}[T \cos (\alpha+\delta)-D]-g \sin y_{\gamma}-\left(\dot{w}_{x} \cos y_{\gamma}+\dot{w}_{h} \sin y_{\gamma}\right)$,

$\dot{y}_{\gamma}=\frac{1}{m y_{\nu}}[T \sin (\alpha+\delta)+L]-\frac{g}{y_{\nu}} \cos y_{\gamma}+\left(\dot{w}_{x} \sin y_{\gamma}-\dot{w}_{h} \cos y_{\gamma}\right)$,

where the state variables

$$
\mathbf{y}(t)=\left[\begin{array}{l}
y_{x}(t) \\
y_{h}(t) \\
y_{\nu}(t) \\
y_{\gamma}(t)
\end{array}\right]
$$

are the horizontal distance $y_{x}$, the altitude $y_{h}$, the relative velocity $y_{\nu}$ and the relative flight path angle $y_{\gamma}$. Moreover, the thrust and aerodynamic forces were defined

$$
\begin{aligned}
& T=\beta T_{\star}, \\
& T_{\star}=a_{0}+a_{1} y_{\nu}+a_{2} y_{\nu}^{2}, \\
& D=\frac{1}{2} C_{D} \rho S y_{\nu}^{2}, \\
& C_{D}(\alpha)=b_{0}+b_{1} \alpha+b_{2} \alpha^{2}, \\
& L=\frac{1}{2} C_{L} \rho S y_{\nu}^{2}, \\
& C_{L}(\alpha)=\left\{\begin{array}{r}
c_{0}+c_{1} \alpha, \text { for } \alpha \leq \alpha_{\star}, \\
c_{0}+c_{1} \alpha+c_{2}\left(\alpha-\alpha_{\star}\right)^{2} \\
\text { for } \alpha_{\star} \leq \alpha \leq \alpha_{\max }
\end{array}\right. \\
& \beta(t)= \begin{cases}\beta_{0}+\dot{\beta}_{1} t, & \text { for } 0 \leq t \leq t_{\beta} \\
1, & \text { for } t_{\beta} \leq t \leq t_{F}\end{cases}
\end{aligned}
$$

where the thrust, drag and lift were denoted as $T, D$ and $L$, respectively. Upon sensing a downdraft, the pilot increases power at a constant rate until reaching the maximum value at time

$$
t_{\beta}=\left(1-\beta_{0}\right) / \dot{\beta}_{0} .
$$

and holds it constant. The windshear is modeled as follows

$$
\begin{aligned}
& w_{x}=A\left(y_{x}\right), \\
& w_{h}=\frac{h}{h_{\star}} B\left(y_{x}\right),
\end{aligned}
$$

where

$$
A\left(y_{x}\right)=\left\{\begin{array}{c}
-50+a y_{x}^{3}+b y_{x}^{4}, \text { for } 0 \leq y_{x} \leq 500 \\
\frac{1}{40}\left(y_{x}-2300\right), \text { for } 500 \leq y_{x} \leq 4100 \\
50-a\left(4600-y_{x}\right)^{3}-b\left(4600-y_{x}\right)^{4} \\
50, \text { for } 4600 \leq y_{x}, \quad \text { for } 4100 \leq y_{x} \leq 4600
\end{array}\right.
$$


TABLE I

THE MODEL PARAMETERS OF A BOEING 727 AIRPLANE [2].

\begin{tabular}{|c|c|}
\hline$t_{F}$ & $40 \mathrm{sec}$ \\
\hline$u_{\max }$ & $3 \mathrm{deg} / \mathrm{sec}$ \\
\hline$\alpha_{\max }$ & $17,2 \mathrm{deg}$ \\
\hline$\rho$ & $0,2203 \times 10^{-2} \mathrm{lb} \mathrm{sec}^{2} \mathrm{ft}^{-4}$ \\
\hline$S$ & $0,1560 \times 10^{4} \mathrm{ft}^{2}$ \\
\hline$g$ & $3,2172 \times 10^{1} \mathrm{ft} \mathrm{sec}^{-2}$ \\
\hline$m g$ & $1,5 \times 10^{5} \mathrm{lb}$ \\
\hline$\delta$ & $2 \mathrm{deg}$ \\
\hline$a_{0}$ & $0,4456 \times 10^{5} \mathrm{lb}$ \\
\hline$a_{1}$ & $-0,2398 \times 10^{2} \mathrm{lb} \mathrm{sec} / \mathrm{ft}$ \\
\hline$a_{2}$ & $0,1442 \times 10^{-1} \mathrm{lb} \mathrm{sec}^{2} \mathrm{ft}^{-2}$ \\
\hline$\beta_{0}$ & 0,3825 \\
\hline$\dot{\beta}_{0}$ & $0,2 \mathrm{sec}^{-1}$ \\
\hline$b_{0}$ & 0,1552 \\
\hline$b_{1}$ & $0,12369 \mathrm{rad}^{-1}$ \\
\hline$b_{2}$ & $2,4203 \mathrm{rad}^{-2}$ \\
\hline$c_{0}$ & 0,7125 \\
\hline$c_{1}$ & $6,0877 \mathrm{rad}^{-1}$ \\
\hline$c_{2}$ & $-9,0277 \mathrm{rad}^{-2}$ \\
\hline$a_{\star}$ & $12 \mathrm{deg}$ \\
\hline$h_{\star}$ & $1000 \mathrm{ft}$ \\
\hline$a$ & $6 \times 10^{-8} \mathrm{sec}^{-1} \mathrm{ft}^{-2}$ \\
\hline$b$ & $-4 \times 10^{-11} \mathrm{sec}^{-1} \mathrm{ft}^{-3}$ \\
\hline$c$ & $-\ln (25 / 30,6) \times 10^{-12} \mathrm{ft}^{-4}$ \\
\hline$d$ & $-8,02881 \times 10^{-8} \mathrm{sec}^{-1} \mathrm{ft}^{-2}$ \\
\hline$e$ & $6,28083 \times 10^{-11} \mathrm{sec}^{-1} \mathrm{ft}-3$ \\
\hline$x_{0}$ & $0 \mathrm{ft}$ \\
\hline$\gamma_{0}$ & $-2,249 \mathrm{deg}$ \\
\hline$h_{0}$ & $600 \mathrm{ft}$ \\
\hline$\alpha_{0}$ & $7,353 \mathrm{deg}$ \\
\hline$\nu_{0}$ & $239,7 \mathrm{ft} / \mathrm{sec}$ \\
\hline$\gamma_{F}$ & $7,431 \mathrm{deg}$ \\
\hline
\end{tabular}

$$
B\left(y_{x}\right)=\left\{\begin{array}{c}
d y_{x}^{3}+e y_{x}^{4}, \text { for } 0 \leq y_{x} \leq 500, \\
-51 \exp \left[-c\left(y_{x}-2300\right)^{4}\right], \\
\quad \text { for } 500 \leq y_{x} \leq 4100, \\
d\left(4600-y_{x}\right)^{3}+e\left(4600-y_{x}\right)^{4}, \\
\quad \text { for } 4100 \leq y_{x} \leq 4600 \\
0, \text { for } 4600 \leq y_{x} .
\end{array}\right.
$$

The model parameters of a Boeing 727 airplane were summarized in the Table I.

The goal of this problem is to avoid having the airplane crash. To achieve this, two important conditions need to be satisfied. An unknown parameter $h_{\text {min }}$ represents the minimum altitude that occurs during the maneuver. Moreover, an additional inequality constraint

$$
y_{h}(t) \geq h_{\min }
$$

was introduced. Then, a new objective function can be constructed

$$
h_{\min } \rightarrow \max
$$

This representation of the optimization problem guarantees that aircraft will be as high above the ground as possible. In the considered task the angle of attack $\alpha(t)$ is treated as the control variable. Moreover, the control variable is subject to the two inequality constraints

$$
u(t)=\alpha(t) \leq \alpha_{\max }
$$

and

$$
|\dot{u}(t)|=|\dot{\alpha}(t)| \leq u_{\max }
$$

The eq. (14) has a character of a variability constraint.

In the presented task five flight phases can be distinguished. Each flight phase is characterized by the appropriate boundary conditions, as well as additional features.

Phase 1: $0=t_{0}^{1} \leq t \leq t_{F}^{1}$

$$
\begin{aligned}
y_{x}\left(t_{0}^{1}\right) & =y_{x_{0}}, \\
y_{\nu}\left(t_{0}^{1}\right) & =y_{\nu_{0}}, \\
y_{h}\left(t_{0}^{1}\right) & =y_{h_{0}}, \\
y_{\gamma}\left(t_{0}^{1}\right) & =y_{\gamma_{0}}, \\
u_{\alpha}\left(t_{0}^{1}\right) & =u_{\alpha_{0}}, \\
y_{x}\left(t_{F}^{1}\right) & =500, \\
A\left(y_{x}\right) & =-50+a y_{x}^{3}+b y_{x}^{4}, \\
B\left(y_{x}\right) & =d y_{x}^{3}+e y_{x}^{4}, \\
\beta(t) & =\beta_{0}+\dot{\beta}_{0} t .
\end{aligned}
$$

Phase 2: $t_{I}^{2} \leq t \leq t_{F}^{2}=t_{\beta}$

$$
\begin{aligned}
y_{x}\left(t_{0}^{2}\right) & =500 \\
A\left(y_{x}\right) & =-50+a y_{x}^{3}+b y_{x}^{4}, \\
B\left(y_{x}\right) & =d y_{x}^{3}+e y_{x}^{4}, \\
\beta(t) & =\beta_{0}+\dot{\beta}_{0} t .
\end{aligned}
$$

Phase 3: $t_{\beta}=t_{I}^{3} \leq t \leq t_{F}^{3}$

$$
\begin{aligned}
y_{x}\left(t_{F}^{3}\right) & =4100, \\
A\left(y_{x}\right) & =\frac{1}{40}\left(y_{x}-2300\right), \\
B\left(y_{x}\right) & =-51 \exp \left(-c\left(y_{x}-2300\right)^{4}\right), \\
\beta(t) & =1 .
\end{aligned}
$$

Phase 4: $t_{0}^{4} \leq t \leq t_{F}^{4}$

$$
\begin{aligned}
y_{x}\left(t_{0}^{4}\right) & =4100, \\
y_{x}\left(t_{F}^{4}\right) & =4600, \\
A\left(y_{x}\right) & =50-a\left(4600-y_{x}\right)^{3}-b\left(4600-y_{x}\right)^{4}, \\
B\left(y_{x}\right) & =d\left(4600-y_{x}\right)^{3}+e\left(4600-y_{x}\right)^{4}, \\
\beta(t) & =1 .
\end{aligned}
$$

Phase 5: $t_{0}^{5} \leq t \leq t_{F}^{5}$

$$
\begin{aligned}
y_{x}\left(t_{0}^{5}\right) & =4600, \\
y_{\gamma}\left(t_{F}^{5}\right) & =y_{\gamma_{F}}, \\
A\left(y_{x}\right) & =50, \\
B\left(y_{x}\right) & =0, \\
\beta(t) & =1 .
\end{aligned}
$$


As one can observe, each stage of the process is described by a system of differential-algebraic equations

$$
\begin{aligned}
\dot{\mathbf{y}}(t) & =F(\mathbf{y}(t), \mathbf{z}(t), \mathbf{u}(t), \mathbf{p}, t) \\
0 & =G(\mathbf{y}(t), \mathbf{z}(t), \mathbf{u}(t), \mathbf{p}, t)
\end{aligned}
$$

where $\mathbf{y}(t) \in \mathcal{R}^{n_{\mathbf{y}}}$ denotes a differential state trajectory, $\mathbf{z}(t) \in \mathcal{R}^{n_{\mathbf{z}}}$ is an algebraic state trajectory, $\mathbf{u}(t) \in \mathcal{R}^{n_{\mathbf{u}}}$ denotes a control function, $\mathbf{p} \in \mathcal{R}^{n_{\mathbf{p}}}$ is a vector of global parameters constant in the time. The indendent variable was time denoted by $t \in \mathcal{R}$. Moreover, two vector-valed functions are considered

$$
\begin{aligned}
& F: \mathcal{R}^{n_{\mathbf{y}}} \times \mathcal{R}^{n_{\mathbf{z}}} \times \mathcal{R}^{n_{\mathbf{u}}} \times \mathcal{R}^{n_{\mathbf{p}}} \times \mathcal{R} \rightarrow \mathcal{R}^{n_{\mathbf{y}}} \\
& G: \mathcal{R}^{n_{\mathbf{y}}} \times \mathcal{R}^{n_{\mathbf{z}}} \times \mathcal{R}^{n_{\mathbf{u}}} \times \mathcal{R}^{n_{\mathbf{p}}} \times \mathcal{R} \rightarrow \mathcal{R}^{n_{\mathbf{z}}}
\end{aligned}
$$

Assumption 2.1: The index of the DAEs system (20) is not greater than 1 .

To solve the flight optimization task presented by eq. (1)-(19), the new optimization-based solving procedure was designed.

\section{ThE NEW OPTIMIZATION-BASED SOLVING PROCEDURE}

The optimization procedure is consisted on two main steps. The first one is based on the multiple shooting method and a control vector parameterization. In the second step - the parameterized model is optimized by a barrier algorithm.

Each phase of the flight was described by a system of differential-algebraic equations (1)-(19). Moreover, each phase was discretized using a modified direct shooting approach, where left-hand side of ODEs are treated also as additional decision variables [7]. Then, with 3 shooting points in each interval, in the considered process $3 \times 5=15$ subintervals were distinguished. Therefore, the time domain

$$
t \in\left[\begin{array}{ll}
t_{0} & t_{f}
\end{array}\right]
$$

was divided according to the indicated 15 subdomains in the following way

$$
t^{i} \in\left[\begin{array}{ll}
t_{0}^{i} & t_{f}^{i}
\end{array}\right], \quad i=1, \cdots, 15,
$$

where

$$
t_{0}=t_{0}^{1}<t_{f}^{1}<\cdots=t_{0}^{15}<t_{f}^{15}=t_{f}
$$

The considered DAE constraints were pointwise discretized as follows

$$
\begin{aligned}
\mathbf{x}_{\dot{\mathbf{y}}^{i}} & =\widetilde{F}^{i}\left(\mathbf{x}_{\mathbf{y}^{i}}, \mathbf{x}_{\mathbf{z}^{i}}, \mathbf{x}_{\mathbf{u}^{i}}, \mathbf{x}_{\mathbf{p}}, t^{i}\right) \\
0 & =\widetilde{G}^{i}\left(\mathbf{x}_{\mathbf{y}^{i}}, \mathbf{x}_{\mathbf{z}^{i}}, \mathbf{x}_{\mathbf{u}^{i}}, \mathbf{x}_{\mathbf{p}}, t^{i}\right)
\end{aligned}
$$

where

$$
\begin{aligned}
\mathbf{x}_{\dot{\mathbf{y}}^{i}} & =\dot{\mathbf{y}}\left(t_{0}^{i}\right) \\
\mathbf{x}_{\mathbf{y}^{i}} & =\mathbf{y}\left(t_{0}^{i}\right) \\
\mathbf{x}_{\mathbf{z}^{i}} & =\mathbf{z}\left(t_{0}^{i}\right) \\
\mathbf{x}_{\mathbf{u}^{i}} & =\mathbf{u}\left(t_{0}^{i}\right) \\
\mathbf{x}_{\mathbf{p}} & =\mathbf{p}
\end{aligned}
$$

and

$$
\begin{aligned}
& \widetilde{F}^{i}: \mathcal{R}^{n_{\mathbf{x}_{\mathbf{y}} i}} \times \mathcal{R}^{n_{\mathbf{x}_{\mathbf{z}} i}} \times \mathcal{R}^{n_{\mathbf{x}_{\mathbf{u}} i}} \times \mathcal{R}^{n_{\mathbf{x}_{\mathbf{p}}}} \times \mathcal{R} \rightarrow \mathcal{R}^{n_{\mathbf{x}_{\mathbf{y}} i}} \\
& \widetilde{G}^{i}: \mathcal{R}^{n_{\mathbf{x} \mathbf{y}^{i}}} \times \mathcal{R}^{n_{\mathbf{x}_{\mathbf{z}^{i}}}} \times \mathcal{R}^{n_{\mathbf{x}_{\mathbf{u}} i}} \times \mathcal{R}^{n_{\mathbf{x}_{\mathbf{p}}}} \times \mathcal{R} \rightarrow \mathcal{R}^{n_{\mathbf{x}_{\mathbf{z}^{i}}}}
\end{aligned}
$$

for $i=1, \ldots, 15$.

Moreover, if

$$
X=\left[\begin{array}{c}
\mathbf{x}_{\dot{\mathbf{y}}^{1}} \\
\vdots \\
\mathbf{x}_{\dot{\mathbf{y}}^{15}} \\
\mathbf{x}_{\mathbf{y}^{1}} \\
\vdots \\
\mathbf{x}_{\mathbf{y}^{15}} \\
\mathbf{x}_{\mathbf{z}^{1}} \\
\vdots \\
\mathbf{x}_{\mathbf{z}^{15}} \\
\mathbf{x}_{\mathbf{u}^{1}} \\
\vdots \\
\mathbf{x}_{\mathbf{u}^{15}}
\end{array}\right]
$$

and with differential-algebraic constraints in the following form

$$
\begin{aligned}
\widetilde{F}^{i}\left(\mathbf{x}_{\mathbf{y}^{i}}, \mathbf{x}_{\mathbf{z}^{i}}, \mathbf{x}_{\mathbf{u}^{i}}, \mathbf{x}_{\mathbf{p}}, t^{i}\right)-\mathbf{x}_{\dot{\mathbf{y}}^{i}} & =0 \\
\widetilde{G}^{i}\left(\mathbf{x}_{\mathbf{y}^{i}}, \mathbf{x}_{\mathbf{z}^{i}}, \mathbf{x}_{\mathbf{u}^{i}}, \mathbf{x}_{\mathbf{p}}, t^{i}\right) & =0
\end{aligned}
$$

for $i=1, \ldots, 15$. Then, the process constraints can be reduced to the general form

$$
\begin{aligned}
& \hat{F}(\mathbf{X})=0 \\
& \hat{G}(\mathbf{X})=0
\end{aligned}
$$


The presented modified direct shooting approach enables us to consider the variability constraints in the following form

$$
y^{L} \leq \dot{\mathbf{y}}(t) \leq y^{U}
$$

directly by

$$
\mathbf{x}_{\dot{y}}^{L} \leq \mathbf{x}_{\dot{y}} \leq \mathbf{x}_{\dot{y}}^{U},
$$

where $L$ and $U$ denote lower and upper bounds, respectively. After that, the appropriate nonlinear optimization problem with a pointwise-continuous differential-algebraic constraints can be considered

$$
\min _{\mathbf{X}} f(\mathbf{X})
$$

subject to

$$
\begin{aligned}
& \hat{F}(\mathbf{X})=0 \\
& \hat{G}(\mathbf{X})=0 \\
& \mathbf{X}_{\dot{\mathbf{y}}} \leq \mathcal{V}(\mathbf{X}) \\
& \mathbf{X}^{L} \leq \mathbf{X} \leq \mathbf{X}^{U}
\end{aligned}
$$

To solve the nonlinear optimization problem with the variability constraints, the barrier method implemented in function fmincon in MATLAB Optimization Toolbox was applied [11]. The obtained results were presented in the next section.

\section{RESUlts OF COMPUTATIONAL SIMULATIONS}

The aircraft state trajectories have been obtained after 1,101 iterations and 170,855 objective function evaluation. The final value of the objective function was equal to $429.6 \mathrm{~m}$. The results of the presented solving procedure were illustrated on the Fig. (2)-(6). The results and shape of the state trajectories are comparable with the solution obtained using Sparse Optimal Control Software - SOCS, developed by The Boeing Company [2].

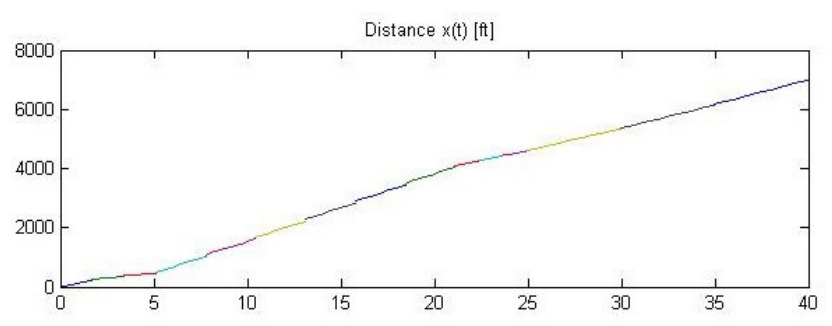

Fig. 2. The state trajectory $y_{x}(t)$.

\section{CONCLUSION}

In the article the modified direct shooting approach for the aircraft landing control problem in the presence of windshear was presented. In the discussed methodology, the values of the state trajectories, as well as left hand-side values of the ODEs, are treated as the additional decision variables. As the results of presented transformation, a large-scale nonlinear optimization problem with pointwise-continuous differential-algebraic

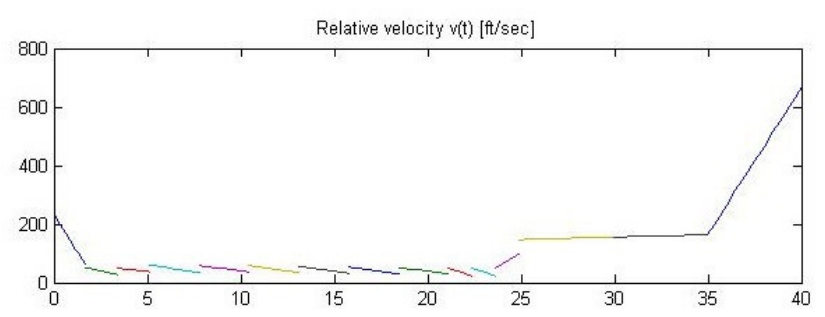

Fig. 3. The state trajectory $y_{v}(t)$.

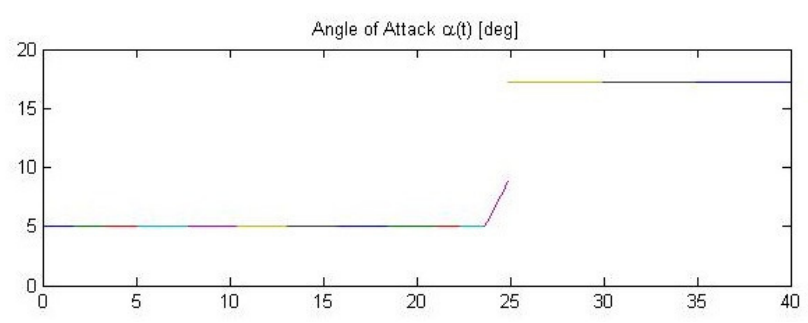

Fig. 4. The control trajectory $\alpha(t)$.

constraints was obtained. To solve the nonlinear optimization task, the barrier algorithm implemented in fmincon function in Matlab environment was used.

The future work should be concentrated on the NLP solver improvements in order to minimize number of the objective function evaluations. Moreover, the presented modifications can be applied in the autonomous takeoff and landing procedures [6], [12], [19].

\section{ACKNOWLEDGMENT}

The work of Paweł Drąg has been supported by the grant „Młoda Kadra”- „Young Staff” 0402/0228/16 at Wrocław University of Science and Technology. The work of Krystyn

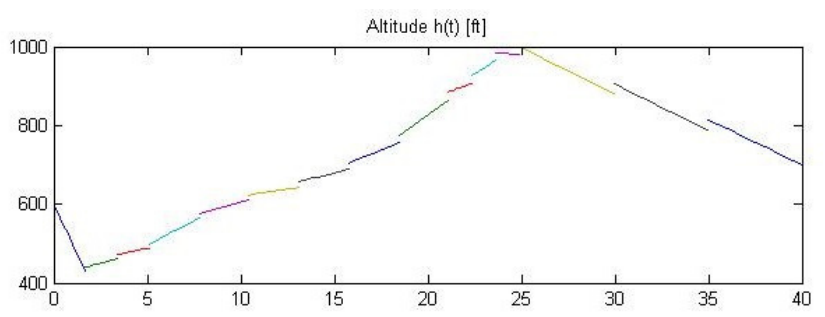

Fig. 5. The state trajectory $y_{h}(t)$.

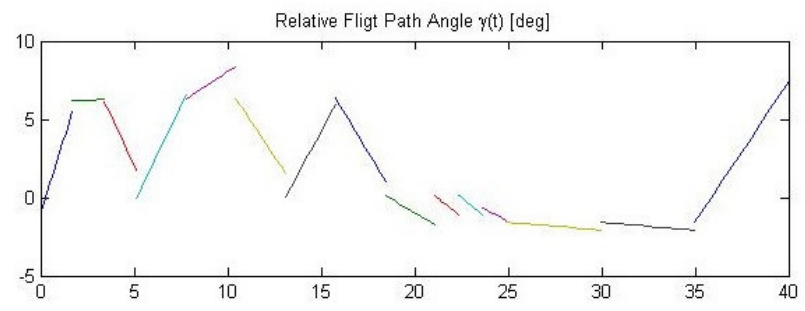

Fig. 6. The state trajectory $y_{\gamma}(t)$. 
Styczeń has been supported by the National Science Center under grant: DEC-2012/07/B/ST7/01216

\section{REFERENCES}

[1] S. Baccari, L. Iannelli, F. Vasca. 2012. A Parallel Algorithm for Implicit Model Predictive Control with Barrier Function. 2012 IEEE International Conference on Control Applications (CCA) Part of 2012 IEEE MultiConference on Systems and Control October 3-5, 2012. Dubrovnik, Croatia. 1405-1410, http://dx.doi.org/10.1109/CCA.2012.6402342

[2] J.T. Betts. 2010. Practical Methods for Optimal Control and Estimation Using Nonlinear Programming. SIAM, Philadelphia, http://dx.doi.org/10.1137/1.9780898718577

[3] R. Bulirsch, F. Montrone, H.J. Pesch. 1991. Abort landing in the presence of windshear as a minimax optimal control problem, part 1: Necessary conditions. Journal of Optimization Theory and Applications. 70:1-23, http://dx.doi.org/10.1007/BF00940502

[4] R. Bulirsch, F. Montrone, H.J. Pesch. 1991. Abort landing in the presence of windshear as a minimax optimal control problem, part 2: Multiple shooting and homotopy. Journal of Optimization Theory and Applications. 70:223-254, http://dx.doi.org/10.1007/BF00940625

[5] P.W. Chan, K.K. Hon. 2016. Observation and Numerical Simulation of Terrain-Induced Windshear at the Hong Kong International Airport in a Planetary Boundary Layer without Temperature Inversions. Advances in Meteorology. Article ID 1454513 , http://dx.doi.org/10.1155/2016/1454513

[6] L.H. De Godoy Patire, N.B.F Silva, K.R.L.J.C Branco. 2016. Data fusion techniques applied to takeoff and landing procedures - A VTOL case study. IEEE Latin America Transactions. 14:3962-3966. http://dx.doi.org/10.1109/TLA.2016.7785919

[7] P. Dra̧g. 2016. Algorytmy sterowania wielostadialnymi procesami deskryptorowymi. Warszawa, Akademicka Oficyna Wydawnicza EXIT, (in polish)

[8] C. Feller, C. Ebenbauer. 2017. A stabilizing iteration scheme for model predictive control based on relaxed barrier functions. Automatica. 80:328339, https://doi.org/10.1016/j.automatica.2017.02.001

[9] K.K. Hon, P.W. Chan. 2014. Terrain-Induced Turbulence Intensity during Tropical Cyclone Passage as Determined from Airborne, Ground-Based, and Remote Sensing Sources. Journal of Atmospheric and Oceanic Technology. 31:2373-2391, http://dx.doi.org/10.1175/JTECH-D-14-00006.1

[10] B. Li, K.L. Teo, G.H. Zhao, G.R. Duan. 2009. An efficient computational approach to a class of minmax optimal con- trol problems with applications. ANZIAM Journal. 51:162-177, http://doi.org/10.1017/S1446181110000040

[11] MathWorks. 2017. Global Optimization Toolbox. User's Guide R2017a.

[12] F.L.L. Medeiros, V.C.F. Gomes, M.R.C. De Aquino, D. Geraldo, M.E.L. Honorato, L.H.M. Dias. 2015. Proceedings - 2015 Brazilian Conference on Intelligent Systems, BRACIS 2015, pp. 333-338, http://doi.org/10.1109/BRACIS.2015.53

[13] A. Miele. 1990. Optimal trajectories and guidance trajectories for aircraft flight through windshears. Proceedings of the 29th IEEE Conference on Decision and Control Part 6 (of 6); Honolulu, HI, USA; 5-7 December 1990. 2:737-746, http://dx.doi.org/10.1109/CDC.1990.203686

[14] C. Moscardini, F. Berizzi, M. Martorella, A. Capria. 2011. Signal spectral modelling for airborne radar in the presence of windshear phenomena. IET Radar, Sonar and Navigation. 5:796-805, http://dx.doi.org/10.1049/iet-rsn.2010.0234

[15] T. Ohtsuka. 2004. A continuation/GMRES method for fast computation of nonlinear receding horizon control. Automatica. 40:563-574, http://doi.org/10.1016/j.automatica.2003.11.005

[16] V.S. Patsko, N.D. Botkin, V.M. Kein, V.L. Turova, M.A. Zarkh. 1994. Control of an aircraft landing in windshear. Journal of Optimization Theory and Applications. 83:237-267, http://dx.doi.org/10.1007/BF02190056

[17] R. Pytlak, R.B. Vinter. 1999. Feasible Direction Algorithm for Optimal Control Problems with State and Control Constraints: Implementation. Journal of Optimization Theory and Applications. 101:623-649, http://dx.doi.org/10.1023/A:1021742204850

[18] C. Shen, B. Buckham, Y. Shi. 2016. Modified C/GMRES Algorithm for Fast Nonlinear Model Predictive Tracking Control of AUVs. IEEE Transactions on Control Systems Technology, http://dx.doi.org/10.1109/TCST.2016.2628803, (to appear)

[19] N.B.F. Silva, E.A. Marconato, K.R.L.J.C. Branco. 2015. AVALON: Definition and modeling of a vertical takeoff and landing UAV. Journal of Physics: Conference Series. Vol. 633, Article number 012125 , http://dx.doi.org/10.1088/1742-6596/633/1/012125

[20] A. Steinboeck, M. Guay, A. Kugi. 2016. Real-Time Nonlinear Model Predictive Control of a Transport-Reaction System. Industrial and Engineering Chemistry Research. 55:7730-7741, http://dx.doi.org/10.1021/acs.iecr.6b00592

[21] Szefostwo Służby Hydrometeorologicznej Sił Zbrojnych RP. 2011. Meteorologia dla pilotów - poradnik. Warszawa, 2011, (in polish)

[22] L. Zhao, X. Yang, H. Gao, P. Shi. 2013. Automatic Landing System Design Using Multiobjective Robust Control. Journal of Aerospace Engineering. 26:603-617, http://dx.doi.org/10.1061/(ASCE)AS.19435525.0000174 